\section{Case Reports in Oncology}

\title{
Paraneoplastic Pemphigus as a First Manifestation of an Intra-Abdominal Follicular Dendritic Cell Sarcoma: Rare Case and Review of the Literature
}

\author{
Reem Akel ${ }^{a} \quad$ Ghina Fakhri $^{\mathrm{a}} \quad$ Rana Salem $^{\mathrm{a}} \quad$ Fouad Boulos $^{\mathrm{b}}$ \\ Khaled Habibc ${ }^{c}$ Arafat Tfaylia
}

aDivision of Hematology and Oncology, Department of Internal Medicine, American University of Beirut Medical Center, Beirut, Lebanon; ${ }^{b}$ Department of Pathology and Laboratory Medicine, American University of Beirut Medical Center, Beirut, Lebanon; 'Specialized Medical Laboratory Al-Mazraa, Beirut, Lebanon

\section{Keywords}

Follicular dendritic cell sarcoma $\cdot$ Paraneoplastic pemphigus $\cdot$ Neoplasm

\begin{abstract}
Background: Follicular dendritic cell sarcoma (FDCS) is an extremely rare malignant tumor caused by proliferation of antigen-presenting follicular dendritic cells. The tumor most commonly arises in lymph nodes, with fewer than $30 \%$ of cases originating in extranodal sites. Case Presentation: We report the case of a 39 -year-old previously healthy male patient who presented with paraneoplastic pemphigus in the setting of a large intraperitoneal mass with no associated lymphadenopathy. The biopsy results showed a cellular proliferation of mildly to moderately atypical oval-to-spindle-shaped cells admixed with a variably dense lymphoplasmacytic infiltrate suggestive of FDCS versus inflammatory myofibroblastic tumor. A pathology review following total resection of the abdominal mass confirmed the diagnosis of FDCS. The tumor cells showed positivity for CD35, CD21, CD45, and CD68, negative ALK staining, and limited smooth muscle actin immunoreactivity. The surgery was complicated by severe
\end{abstract}


pneumonia and acidosis, resulting in a prolonged ICU stay and death. Conclusion: It is essential to maintain a high index of suspicion for FDCS and include it in the differential diagnosis of a spindle cell neoplasm, especially in the setting of paraneoplastic pemphigus. The histological and immunohistochemical features should be sufficient to confirm the diagnosis of FDCS.

\section{Introduction}

Follicular dendritic cell sarcoma (FDCS) is an extremely rare malignant tumor caused by proliferation of antigen-presenting follicular dendritic cells [1]. It was first described in 1986 by Monda et al. [2], and has since been reported in approximately 343 patients worldwide [3]. The tumor most commonly arises in lymph nodes, especially in the cervical, mediastinal, or axillary areas, and presents as slow-growing painless lymphadenopathy. Fewer than $30 \%$ of cases originate in extranodal sites [1]. The disease's exact etiology and pathogenesis have not yet been elucidated. We herein report a case of paraneoplastic pemphigus (PNP) as the first manifestation of an intraperitoneal FDCS with progressive clinical deterioration following surgery.

\section{Case Presentation}

This is the case of a 39-year-old previously healthy male patient with a 20-pack-year smoking history who presented to another institution for a few days' history of oral ulcers that did not respond to oral antifungal (fluconazole) or antiviral (valacyclovir hydrochloride) treatment. He initially denied having any fever, chills, night sweats, decreased appetite, or weight loss. Ten days following presentation, he developed diffuse pemphigoid lesions on the chest, abdomen, and arms (Fig. 1). Blood tests at the time revealed isolated neutropenia. A follow-up CT scan of the chest, abdomen, and pelvis showed a large $18 \times 8.9 \mathrm{~cm}$ necrotic irregular mesenteric mass with no associated retroperitoneal or femoral-inguinal lymphadenopathy. A needle core biopsy of the mass was not conclusive (Fig. 2).

The patient presented to our institution with febrile neutropenia (WBC count: $900 / \mathrm{mm}^{3}$ ) and decreased oral intake due to diffuse oral ulcers along with diffuse cutaneous pemphigoid lesions. Viral serology for hepatitis B, hepatitis C, and HIV along with EBV PCR was negative. Bone marrow aspirate and biopsy showed $80 \%$ marrow cellularity with markedly left-shifted myelopoiesis, mild dysmegakaryopoiesis, and mild reactive plasmacytosis. There was no evidence of an epithelial or mesenchymal tumor, lymphoproliferative disorder, or acute leukemia. Repeated abdominal biopsy and review of the previous pathology demonstrated a cellular proliferation of mildly to moderately atypical oval-to-spindle-shaped cells admixed with a variably dense lymphoplasmacytic infiltrate. The architecture was fascicular to vaguely storiform, and the stroma was mildly myxoid to collagenous. Tumor cells stained positive for vimentin, CD21, and CD68, and weakly for CD45. They were focally positive for S100 protein, CD34, and SMA (smooth muscle actin), and negative for ALK, CD117, DOG-1, CD3, CD20, CD23, and EBER. Mitotic figures were rare (Fig. 3).

The clinical, morphologic, and immunohistochemical features of this tumor raised differential diagnostic considerations of FDCS versus inflammatory myofibroblastic tumor, favoring the former based on positivity for CD21, CD45, and CD68, negative ALK staining, and limited SMA immunoreactivity. 
The patient was started on high-dose steroids (methylprednisolone $1 \mathrm{mg} / \mathrm{kg}$ intravenously once per day), which contributed to improvement of his oral and skin lesions. He also received granulocyte colony-stimulating factor injections while planning for surgical resection.

An external expert pathology review following total resection of the abdominal mass confirmed the diagnosis of FDCS. The immunohistochemical panel performed by the consultant pathologist showed a profile similar to the one obtained at our pathology laboratory, in addition to diffuse positivity for CD35, which confirmed the diagnosis.

The surgery was complicated by severe pneumonia and acidosis, resulting in a prolonged ICU stay and death.

\section{Discussion}

FDCS is a rare malignant tumor arising from dendritic cells localized within lymphoid follicles. It most commonly affects young-to-middle-aged adults (mean age 43 years) with equal predilection for men and women [1]. A review of the literature suggests that FDCS preferentially affects certain ethnicities in specific geographic locations, particularly East Asia, where many of the cases have been reported [4-8]. No major risk factors were identified to be involved in the occurrence of FDCS.

As expected, this tumor most commonly arises in lymph nodes, with fewer than one-third of cases arising in extranodal sites. Extranodal disease mostly occurs in lymph-rich areas of the head and neck (tonsils and nasopharynx) and, less commonly, intra-abdominal organs [1]. Approximately $12 \%$ of FDCS are associated with EBV, especially when occurring in the liver [8]. Our case is among the few reported cases of intraperitoneal FDCS, in the setting of negative EBV PCR.

Our patient presented with PNP, a life-threatening bullous autoimmune disease that is characterized by severe stomatitis and polymorphous skin eruptions [9]. A review of 163 cases of PNP reported between 1990 and 2003 found that $84 \%$ of the cases were associated with hematologic malignancies (non-Hodgkin lymphoma [39\%], chronic lymphocytic leukemia [18\%], Castleman's disease [18\%], thymoma [6\%], Waldenström's macroglobulinemia [1\%], Hodgkin's lymphoma [1\%], and monoclonal gammopathy [1\%]). The remaining $16 \%$ were associated with nonhematologic neoplasms (epithelial-origin carcinoma [9\%], mesenchymal-origin sarcoma [6\%], and melanoma [1\%]) [10]. The bone marrow biopsy in our case did not reveal any evidence of a hematological malignancy. To date approximately 14 cases of PNP associated with soft tissue sarcoma have been reported, only 4 of which were FDCS [5]. Lee et al. [5] described a case of PNP with lesions exclusively limited to mucosal membranes without skin involvement that developed shortly following the resection of an FDCS. Chow et al. [4] reported a case of FDCS of the anterior mediastinum presenting initially as PNP in a 62year-old man.

The histologic appearance and specific immunohistochemical staining pattern is considered the gold standard for the diagnosis of FDCS. Histology characteristically demonstrates a proliferation of spindle-to-ovoid-shaped cells arranged in sheets, fascicles, or whorls in a storiform pattern with interspersed lymphocytes and a low mitotic rate [1, 8]. Typically, the neoplastic cells of FDCS stain positively for CD21, CD35, and CD23, with variable expression of vimentin, CD68, and S100 protein $[1,11]$. The immunophenotypic profile in our case was similar to that previously reported in other studies. 
Poor prognostic factors for FDCS include extensive necrosis, a large tumor size ( $>6 \mathrm{~cm})$, cytologic atypia, an intra-abdominal location, and a high proliferative index (mitotic count $>5$ mitoses /10 high-power fields), all of which increase the likelihood of recurrence, metastasis, and death. An intra-abdominal location was the single most important unfavorable prognostic factor $[8,12]$. Our patient had several of these features, which may explain the tumor's aggressive clinical course.

\section{Conclusion}

It is essential to maintain a high index of suspicion for FDCS and include it in the differential diagnosis of a spindle cell neoplasm with admixed chronic inflammatory cells, especially in the setting of PNP. The clinical course, although variable, can potentially be grave with associated significant morbidity and mortality.

\section{Acknowledgments}

We would like to acknowledge Dr. Christopher Fletcher, the consultant pathologist, who performed the CD35 staining and helped confirm the diagnosis.

\section{Statement of Ethics}

The authors have no ethical conflicts to disclose.

\section{Disclosure Statement}

None of the authors have any conflict of interest to declare.

\section{Funding Sources}

No funding was provided for this case report.

\section{Author Contributions}

All authors contributed to the literature search, data collection (including figures), and manuscript writing.

\section{References}

1 Chen T, Gopal P. Follicular dendritic cell sarcoma. Arch Pathol Lab Med. 2017 Apr;141(4):596-9.

2 Monda L, Warnke R, Rosai J. A primary lymph node malignancy with features suggestive of dendritic reticulum cell differentiation. A report of 4 cases. Am J Pathol. 1986 Mar;122(3):562-72. 
3 Bouriga R, Abdessaied N, Hochlef M, Mallat N, Mahjoub M, Sriha B et al. Retroperitoneal follicular dendritic cell sarcoma in a young woman: diagnosis and treatment challenges. Curr Probl Cancer. 2018. [Epub ahead of print]

4 Chow SC, Yeung EC, Ng CS, Wong RH, Fai To K, Wan IY. Mediastinal follicular dendritic cell sarcoma with paraneoplastic pemphigus. Asian Cardiovasc Thorac Ann. 2015 Jul;23(6):732-4.

5 Lee SE, Kim HR, Hashimoto T, Kim SC. Paraneoplastic pemphigus developed shortly after resection of follicular dendritic cell sarcoma. Acta Derm Venereol. 2008;88(4):410-2.

6 Liu KL, Shen JL, Yang CS, Chen YJ. Paraneoplastic pemphigus as the first manifestation of follicular dendritic cell sarcoma. J Dtsch Dermatol Ges. 2014 Jan;12(1):68-71.

7 Su Z, Liu G, Liu J, Fang T, Zeng Y, Zhang H et al. Paraneoplastic pemphigus associated with follicular dendritic cell sarcoma: report of a case and review of literature. Int J Clin Exp Pathol. 2015 Oct;8(10):11983-94.

8 Chan JK, Fletcher CD, Nayler SJ, Cooper K. Follicular dendritic cell sarcoma. Clinicopathologic analysis of 17 cases suggesting a malignant potential higher than currently recognized. Cancer. 1997 Jan;79(2):294-313.

9 Anhalt GJ, Kim SC, Stanley JR, Korman NJ, Jabs DA, Kory M et al. Paraneoplastic pemphigus. An autoimmune mucocutaneous disease associated with neoplasia. N Engl J Med. 1990 Dec;323(25):1729-35.

10 Kaplan I, Hodak E, Ackerman L, Mimouni D, Anhalt GJ, Calderon S. Neoplasms associated with paraneoplastic pemphigus: a review with emphasis on non-hematologic malignancy and oral mucosal manifestations. Oral Oncol. 2004 Jul;40(6):553-62.

11 Soriano AO, Thompson MA, Admirand JH, Fayad LE, Rodriguez AM, Romaguera JE et al. Follicular dendritic cell sarcoma: a report of 14 cases and a review of the literature. Am J Hematol. 2007 Aug;82(8):725-8.

12 Leger S, Picard D, Ingen-Housz-Oro S, Arnault JP, Aubin F, Carsuzaa F et al. Prognostic factors of paraneoplastic pemphigus. Arch Dermatol. 2012 Oct;148(10):1165-72.

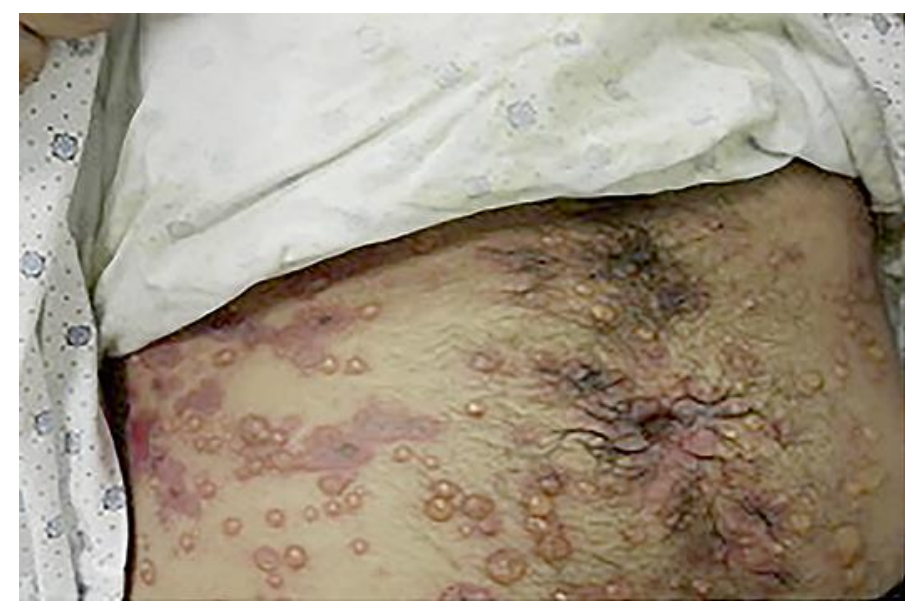

Fig. 1. Diffuse pemphigoid lesions over the abdomen. 


\section{Case Reports in Oncology}
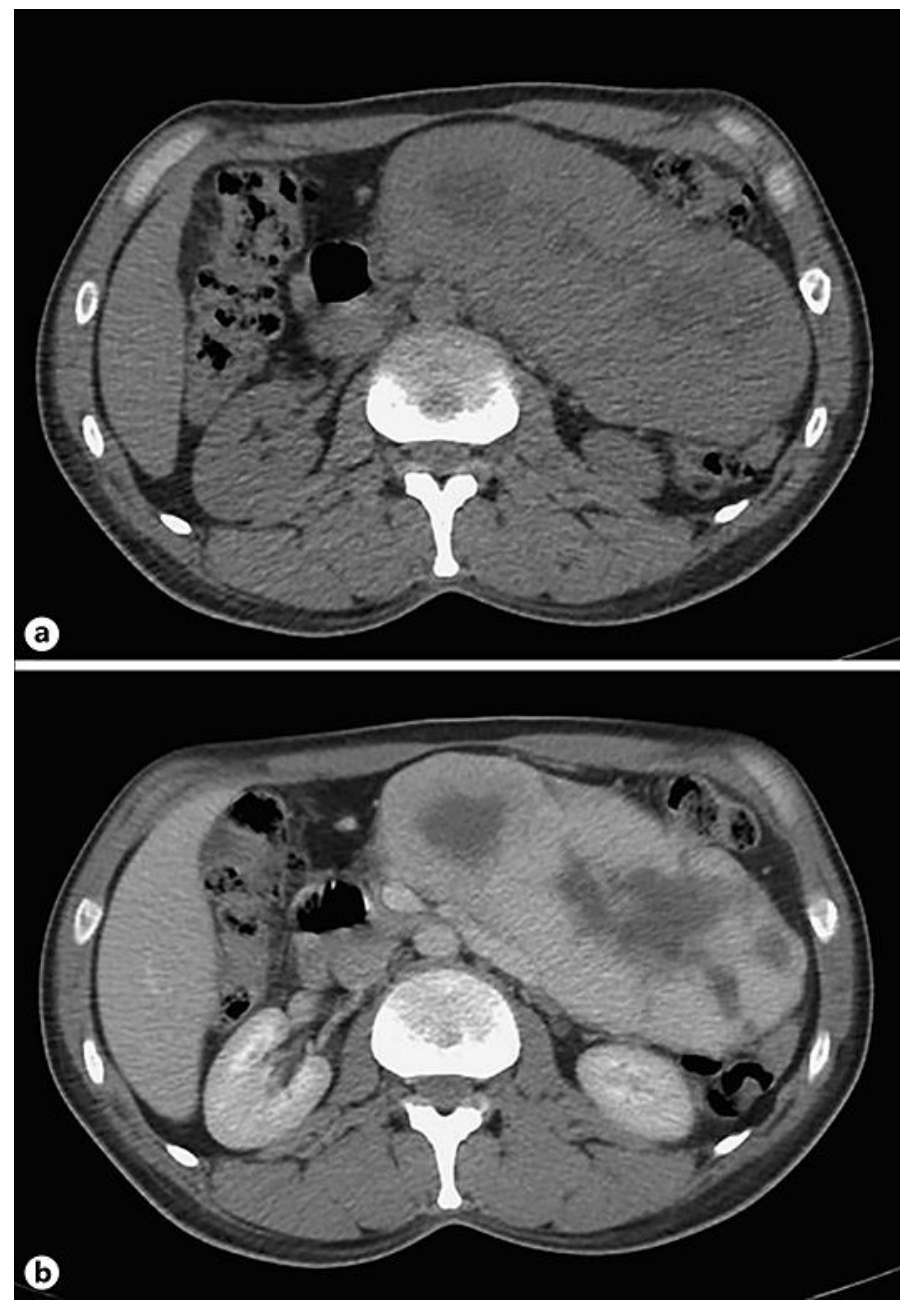

Fig. 2. CT scan of the abdomen and pelvis without (a) and with IV contrast (b). A large lobulated enhancing soft tissue mesenteric mass with necrotic areas in the left upper quadrant of the abdomen crossing the midline can be seen. The mass is compressing the adjacent organs (colon, spleen, and left kidney), displacing the aorta to the right and compressing the mesenteric vessels. 


\section{Case Reports in Oncology}
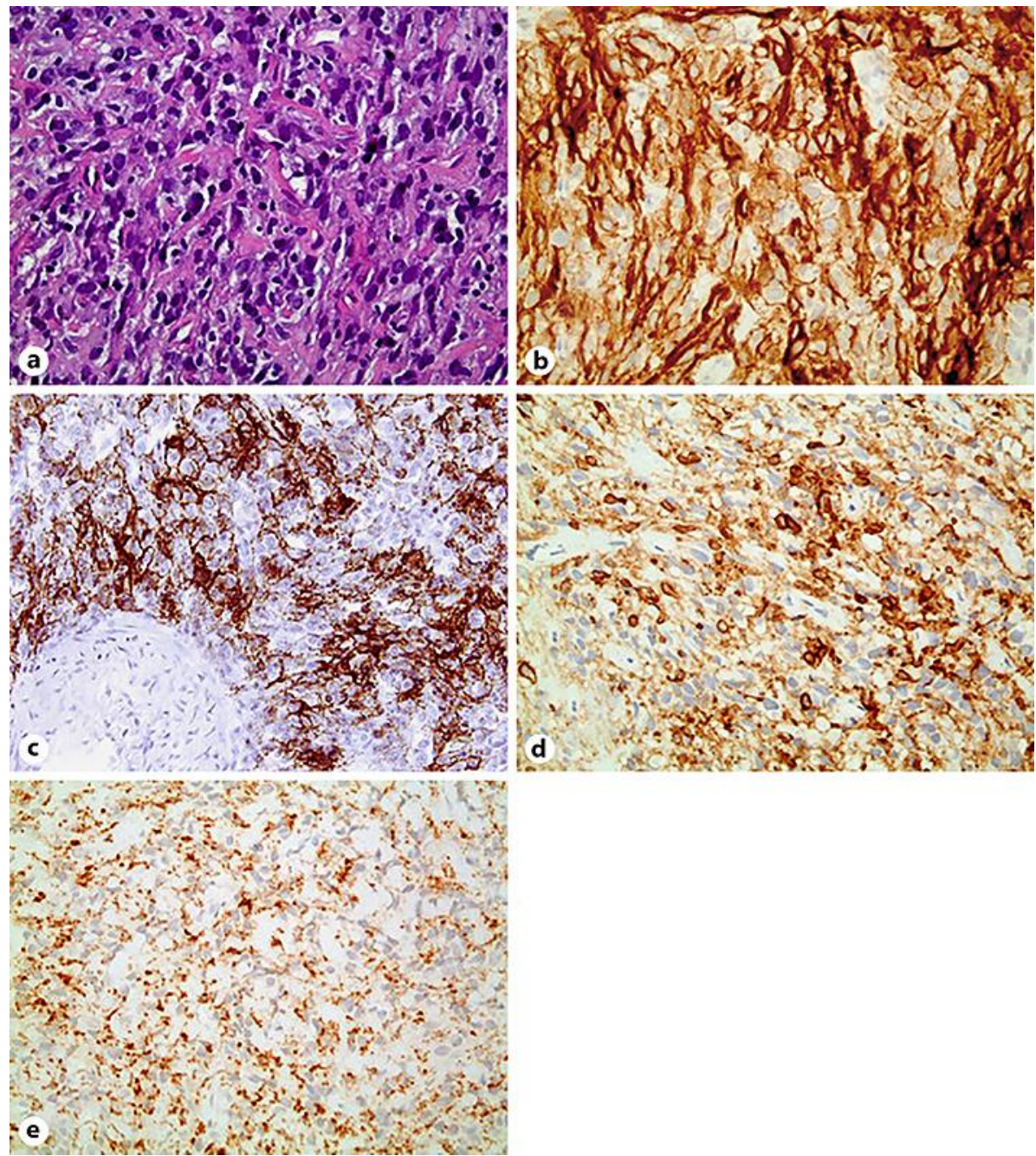

Fig. 3. a Follicular dendritic cell sarcoma composed of a cellular proliferation of mildly to moderately atypical oval-to-spindle-shaped cells admixed with a variably dense lymphoplasmacytic infiltrate (H\&E staining, high power). Immunohistochemical markers of the patient's specimen: CD21 positive (b), CD35 positive (c), weakly CD45 positive (d), and weakly CD68 positive (e). 